\title{
openheart Asymptomatic left ventricular dysfunction with long-term clozapine treatment for schizophrenia: a multicentre cross-sectional cohort study
}

\author{
V Chow, ${ }^{1,2}$ T Yeoh, ${ }^{1,3}$ A C C Ng, ${ }^{1}$ T Pasqualon, ${ }^{4}$ E Scott, ${ }^{8}$ J Plater, ${ }^{4}$ B Whitwell, ${ }^{5}$ \\ D Hanzek, ${ }^{3}$ T Chung, ${ }^{1}$ L Thomas, ${ }^{6}$ D S Celermajer, ${ }^{7}$ L Kritharides ${ }^{1,2}$
}

To cite: Chow V, Yeoh T, $\mathrm{Ng} \mathrm{ACC}$, et al. Asymptomatic left ventricular dysfunction with long-term clozapine treatment for schizophrenia: a multicentre cross-sectional cohort study. Open Heart 2014;1:e00030.

doi:10.1136/openhrt-2013000030

Received 20 December 2013 Revised 15 January 2014 Accepted 30 January 2014

CrossMark

For numbered affiliations see end of article.

Correspondence to

Professor Leonard

Kritharides; leonard.

kritharides@sydney.edu.au

\section{ABSTRACT}

Objectives: Patients with schizophrenia treated with clozapine are at risk of acute myocarditis and dilated cardiomyopathy. However, there are no data on the prevalence of subclinical cardiomyopathy or its associations.

Methods: 100 consecutive patients with schizophrenia treated with clozapine for $>1$ year and without a history of cardiac pathology (group 1), 21 controls with a history of schizophrenia treated with non-clozapine antipsychotics for $>1$ year (group 2) and 20 controls without schizophrenia (group 3) were studied. Comprehensive evaluation by clinical examination, ECG, transthoracic echocardiography including left ventricular ejection fraction (LVEF) and global longitudinal strain (GLS) and biochemical profiles were performed.

Results: Patients with schizophrenia were of similar age, but had higher body mass index (BMI), rates of smoking and hyperlipidaemia than controls. Patients with schizophrenia had received clozapine or nonclozapine antipsychotics for a mean duration of $6.8 \pm 5.3$ and $9.7 \pm 6.1$ years, respectively. Patients taking clozapine demonstrated globally impaired LVEF (58.3\%: group 1 vs $62.2 \%$ : group 2 vs $64.8 \%$ : group 3 , $\mathrm{p}<0.001)$ and GLS (-16.7\%: group 1 vs $-18.6 \%$ : group 2 vs $-20.2 \%$ : group 3, $p<0.001)$. Moreover, LVEF was $<50 \%$ in $9 / 100(9 \%)$ patients receiving clozapine and in non-clozapine schizophrenia patients or healthy controls, but this was not statistically significantly different (analysis of covariance, $p=0.19$ ). Univariate analysis in patients taking clozapine found that impaired LV was not predicted by high-sensitivity troponin $\mathrm{T}$, but was associated with features of the metabolic syndrome (including increased triglycerides, low high-density lipoprotein cholesterol (HDL-C), high-sensitivity C reactive protein and $\mathrm{BMI})$, elevated neutrophil count, elevated heart rate, smoking and N-terminal probrain natriuretic peptide. In patients taking clozapine, multivariable analysis identified elevated neutrophil count and low HDL-C as the only independent predictors of impaired GLS.

Conclusions: Asymptomatic mild LV impairment is common in patients with schizophrenia receiving long-term clozapine treatment and is associated with neutrophilia and low HDL-C.
STRENGTHS AND LIMITATIONS OF THIS STUDY:

- This is the first comprehensive study to assess the prevalence of asymptomatic myocardial dysfunction utilising echocardiography in patients with schizophrenia on long-term clozapine treatment.

- Our study has shown asymptomatic left ventricular dysfunction (with impairment in Simpson's EF and global longitudinal strain) is more common than previously recognised in this patient group.

- The degree of left ventricular dysfunction is independently associated with markers of inflammation including elevated neutrophil count and low high-density lipoprotein (HDL-C).

- The study is cross-sectional in design without prospective data before the commencement of treatment for schizophrenia.

- Future studies will need to determine if the asymptomatic changes in left ventricular function we have observed relate to long term outcome.

\section{INTRODUCTION}

Clozapine is an atypical antipsychotic which is effective in the treatment of refractory schizophrenia. It is particularly useful in patients who do not respond to conventional therapy or are intolerant of other agents. ${ }^{1}$ It achieves greater reductions in suicidal behaviour compared with other antipsychotics in treatment-refractory schizophrenia. ${ }^{2}$ A recent population study concluded that clozapine significantly lowers all-cause mortality relative to other antipsychotic medications. ${ }^{3}$

Despite its efficacy, clozapine use can be associated with significant side effects. These include haematological consequences such as agranulocytosis, neutropenia and leucocytosis, ${ }^{4}$ and cardiac side effects such as acute myocarditis, persistent tachycardia and cardiomyopathy. ${ }^{5}$ Postmarketing experience suggests that 
leucocytosis and neutrophilia occur in up to $10 \%$ of patients receiving clozapine. ${ }^{6}$ Although the incidence of clinically severe clozapine-induced cardiomyopathy is reported as 51.5 per 100000 patient-years, ${ }^{7}$ this is likely to be an underestimate, as cardiomyopathy can be mild. Moreover, the predictors of cardiomyopathy after longterm clozapine treatment are unclear. This is important, as guidelines advocating annual clozapine review and management of clozapine treatment based on cardiac findings have not been universally agreed on, and could be strengthened by improved understanding of the cardiac response to long-term antipsychotic use.

Traditional evaluation of cardiac function has involved assessment of left ventricular ejection fraction (LVEF) by transthoracic echocardiogram (TTE) or gated heart pool scan. Impaired LVEF is associated with adverse long-term prognosis in a wide range of clinical settings. ${ }^{8}$ Recently, two-dimensional (2D) speckle-tracking strain and strain rate measurement by TTE have been introduced as quantitative measurement techniques that detect subtle changes in left ventricular (LV) function, not identified by measurements of traditional parameters such as LVEF. $^{9}$ The superior sensitivity of $2 \mathrm{D}$ speckle-tracking strain and strain rate for LV abnormalities has been validated in patients with congenital heart disease, those with diabetic and hypertensive heart disease $^{10}$ and chemotherapy-induced cardiomyopathy. ${ }^{11}$ Furthermore, it provides stronger prognostic information and appears to be superior to LVEF and wall motion score indices for risk stratification after acute myocardial infarction. ${ }^{12}$ To the best of our knowledge, there have been no systematic studies assessing myocardial strain in patients on long-term clozapine treatment, and the prevalence of asymptomatic myocardial dysfunction in this population is unknown.

The primary aim of our study was, therefore, to undertake clinical, ECG, echocardiographic and biochemical assessment of patients with schizophrenia on long-term treatment with clozapine with no cardiac symptoms and to assess for cardiac dysfunction. We also studied two control groups (with schizophrenia but not receiving clozapine and healthy controls). Our secondary aim was to investigate whether inflammation, tachycardia or other clinical or biochemical parameters might independently predict the extent of LV dysfunction in these patients.

\section{METHODS}

\section{Study population}

From January 2010 to December 2012, consecutive patients on long-term clozapine treatment were recruited from outpatient cardiac clinics established for the routine care of psychiatric patients at two separate hospitals (Concord Repatriation General Hospital and Canterbury Hospital). Two separate control groups were recruited for comparison. The first control group comprised 20 healthy volunteers matched for age and gender who did not have schizophrenia and were not receiving clozapine. The second control group, comprised 21 patients with schizophrenia who had never received clozapine treatment, were currently receiving long-term non-clozapine antipsychotics and had similar baseline characteristics to the patients with clozapine.

Patients were excluded from the analysis if they were: (1) prescribed clozapine or non-clozapine antipsychotic treatment for less than 1 year; (2) were deemed noncompliant with antipsychotic medications based on psychiatric reviews and serum clozapine levels for patients receiving this drug; (3) had a known history of heart disease or (4) had recently been diagnosed with ischaemic heart disease in the course of cardiac screening at the clinics.

All patients with schizophrenia met Diagnostic and Statistical Manual of Mental Disorders, Fourth Edition (DSM-IV) diagnostic criteria for a schizophrenic disorder according to the guidelines of the American Psychiatric Association. ${ }^{13}$ Patients prescribed clozapine had previously shown insufficient clinical response to other antipsychotic treatments or developed intolerable side effects. All patients with abnormal ECG and/or symptoms suggestive of cardiac ischaemia underwent further functional testing (exercise stress echocardiography or myocardial perfusion technetium sestamibi scans) to exclude cardiac ischaemia.

\section{ECG and echocardiography}

Twelve-lead ECG was performed on each participant in accordance with standardised protocols. TTE was performed with participants in the left lateral decubitus position (Vivid7/Vivid Q, GE Medical Systems, Norway). All images were acquired from standard echocardiographic views in accordance with the American Society of Echocardiography recommendations. ${ }^{14}$ All images were stored digitally and analysed offline using commercially available software (GE EchoPac V.7.0.1, Norway). TTE was performed by a single operator at each site and all analyses were performed offline in batches on anonymised images by a single analyst (VC) blinded to clinical details as well as the group of each participant.

The cardiac chambers were analysed by conventional 2D parameters, as well as measurement of longitudinal function included strain and strain rates. All measurements were averaged over three consecutive cardiac cycles. The LVEF was measured by linear (M-mode-derived EF, fractional shortening) and volumetric (Simpson's biplane) methods, and impaired cardiac function was defined as Simpson's LVEF $<50 \%$, consistent with published guidelines. ${ }^{14}$ Global LV function were also assessed using the modified Tei index. ${ }^{15}$ The modified Tei index is a validated measure of global ventricular function incorporating systolic and diastolic function. ${ }^{16}$ Conventional 2D parameters included: ratio of right ventricular (RV) and LV end-diastolic diameters; ratio of $\mathrm{RV}: \mathrm{LV}$ end-diastolic areas; $\mathrm{RV}$ ejection area ( (RV end-diastolic area-RV end-systolic area)/RV end- 
diastolic area); ratio of right atrial (RA) and left atrial end-systolic areas and the RV-RA pressure derived from the tricuspid regurgitant envelope obtained using continuous-wave Doppler. ${ }^{14} \mathrm{RV}$ longitudinal function was assessed using the tricuspid annular plane systolic excursion. ${ }^{17}$ All areas were derived by planimetry from the apical four-chamber view.

Images were acquired for postprocessing and derivation of strain and strain rate (EchoPac V.7.0.1, Norway) as described previously and images were obtained at a rate of 60-80 fps. ${ }^{18}$ Global LV longitudinal strain (GLS) was calculated as an average of all $18 \mathrm{LV}$ segments. Similarly, segmental and global systolic and early (E strain rate) and late (A strain rate) diastolic strain rate was measured. Interobserver and intraobserver variability was performed in a subset of patients $(n=20)$.

\section{Biochemical analyses}

Peripheral venous blood samples were collected, and biochemical analyses included fasting lipid profile (total cholesterol, triglycerides, high-density lipoprotein cholesterol (HDL-C) and low-density lipoprotein cholesterol (LDL-C)), electrolytes, liver and thyroid function tests. Creatine kinase (CK), high-sensitivity troponin $\mathrm{T}$ (HsTropT) and N-terminal probrain natriuretic peptide (NT-proBNP). Measurements of systemic inflammation included full blood count and its differentials (neutrophil, lymphocyte and eosinophil counts) and highsensitivity $\mathrm{C}$ reactive protein (hsCRP).

\section{Statistical analysis}

All continuous variables are expressed as mean and 95\% CI, unless otherwise stated, and categorical data were given as frequencies and percentages. Comparisons between three groups were performed by analysis of covariance (ANCOVA) and post hoc Tukey-Kramer's test with Bonferroni correction. Pearson or Spearman correlation coefficient analysis was used to assess associations between GLS and measured parameters. Multivariable backward selection linear regression analysis was performed to identify independent associations of GLS. We included all variables with a univariate $p$ value of $<0.10$ in the multivariate model to ensure capture of all independent predictors of GLS. Colinearity between variables was tested using variance inflation factor calculation. Based on our own preliminary data, and literature on other examples of subclinical cardiac toxicity, ${ }^{19}{ }^{20}$ we anticipated a $10 \%$ relative difference in GLS between patients with schizophrenia. In order to detect such a difference between patients receiving clozapine and the two control groups with a statistical power of $90 \%$, a type I error risk of $5 \%$ and an estimated SD of $2.5 \%$, a minimum of 17 patients were estimated to be needed in each group. Assuming a $15 \%$ rate of incomplete measurements, we planned for a minimum of 20 patients for each group in the study. Patients receiving clozapine were recruited consecutively over the recruitment period until 20 consecutive patients in the non-clozapine group were recruited. Analysis was performed using SPSS V.15.0 (SPSS Inc, Chicago, Illinois, USA). A two-tailed probability value $<0.05$ was considered statistically significant.

\section{RESULTS \\ Baseline characteristics}

A total of 141 participants participated in the study. We recruited 100 patients receiving clozapine, 21 patients receiving non-clozapine treatment and 20 healthy participants. Table 1 summarises the clinical characteristics of the participants. The mean duration of antipsychotic treatment for the clozapine and non-clozapine schizophrenia cohorts was 6.8 years (95\% CI 1.7 to 16.3 ) and 9.7 years (1.6 to 22.4), respectively. Among patients receiving non-clozapine antipsychotics, 18 patients were receiving atypical antipsychotic medications namely olanzapine (7), quetiapine (4), paliperidone (3), risperidone (2), aripiprazole (1) and amisulpride (1), while 3 patients were receiving typical antipsychotic medications (zuclopenthixol). Analysis of variance of the three groups showed significant differences with respect to resting heart rate (clozapine vs non-clozapine vs controls: 93 (76-115) vs 76 (57-99) vs 70 (61-88) bpm, $\mathrm{p}<0.001)$, body mass index (29.6 (19.2-42.5) vs 29.4 $(19.6-46.7)$ vs $\left.23.5(19.4-26.8) \mathrm{kg} / \mathrm{m}^{2}, \mathrm{p}=0.02\right)$ and history of smoking ( $57 \%$ vs $53 \%$ vs $10 \%, \mathrm{p}<0.001)$ and hypercholesterolaemia ( $30 \%$ vs $29 \%$ vs $0 \%, \mathrm{p}=0.02$ ). On post hoc Tukey analysis, the patients receiving clozapine and non-clozapine drugs were well matched with no significant differences in any baseline characteristics. In total, 11 patients (9 patients receiving clozapine and 2 patients receiving non-clozapine drugs) had abnormal resting ECG (7) or cardiac symptoms (4) potentially suggestive of cardiac ischaemia. Of those with abnormal resting ECGs, five patients had diffuse $T$ wave flattening, one patient was noted to be in ventricular bigeminy and one patient had lateral $\mathrm{T}$ wave inversion associated with ST segment depression. All 11 patients had myocardial ischaemia excluded by stress echocardiography or by myocardial perfusion scan.

Following the initial recruitment of participants, there were two subsequent sudden deaths among patients receiving clozapine treatment, and in both patients there were normal HsTropT and NT-proBNP levels at the time of recruitment. One of the deaths was presumed to be due to drug overdose. The other was due to an acute myocardial infarction confirmed on autopsy.

\section{Biochemical analysis}

Patients on clozapine treatment had significantly higher levels of inflammatory markers (white cell count: 8.2 $(4.4-12.9)$ vs $7.4(3.9-13.8)$ vs $6.5(4.3-9.6) \times 10^{9} / \mathrm{L}$, $\mathrm{p}=0.02$; neutrophil count: $5.4(2.5-10.1)$ vs $4.3(1.6-8.2)$ vs $3.6(2.1-5.6) \times 10^{9} / \mathrm{L}, \mathrm{p}=0.001$; neutrophil : lymphocyte ratio: $2.9(1.1-6.7)$ vs $1.9(0.7-5.2)$ vs $1.7(0.9-4.6)$, $\mathrm{p}=0.001$ and hsCRP $4.3(0.4-12.9)$ vs $3.4(0.1-12.0)$ vs 
Table 1 Baseline characteristics

\begin{tabular}{|c|c|c|c|c|}
\hline & Clozapine $(n=100)$ & Non-clozapine $(n=21)$ & Controls $(n=20)$ & p Value \\
\hline Age (years) & $40 \pm 12$ & $37 \pm 14$ & $43 \pm 13$ & 0.33 \\
\hline \multicolumn{5}{|l|}{ Gender } \\
\hline Male & $54(54)$ & $15(72)$ & $13(65)$ & 0.28 \\
\hline \multicolumn{5}{|l|}{ Schizophrenia } \\
\hline Duration of illness (years) & $16.5 \pm 9.7$ & $11.8 \pm 8.0$ & - & \\
\hline Family history of mental illness & $11(11)$ & $5(24)$ & 0 & 0.06 \\
\hline \multicolumn{5}{|l|}{ Antipsychotic treatment } \\
\hline Duration of treatment (years) & $6.8 \pm 5.3$ & $9.7 \pm 6.1$ & & \\
\hline Dosage at time of study (mg/day) & $372 \pm 155$ & - & & \\
\hline Body mass index $\left(\mathrm{kg} / \mathrm{m}^{2}\right)$ & $29.6 \pm 7.0$ & $29.4 \pm 7.4$ & $23.5 \pm 2.5$ & $0.02^{*} \dagger \ddagger$ \\
\hline \multicolumn{5}{|l|}{ Comorbidities } \\
\hline Alcohol excess & $9(9)$ & $2(10)$ & 0 & 0.38 \\
\hline \multicolumn{5}{|l|}{ Heart failure } \\
\hline Previous history of heart failure & 0 & $1(5)$ & 0 & 0.06 \\
\hline NYHA Class $>1$ & 0 & $1(5)$ & 0 & 0.06 \\
\hline Stroke & $1(1)$ & 0 & 0 & 0.82 \\
\hline \multicolumn{5}{|l|}{ Risk factors for IHD } \\
\hline Diabetes & $23(23)$ & $5(24)$ & $2(10)$ & 0.42 \\
\hline Family history of IHD & $24(24)$ & $3(14)$ & $2(10)$ & 0.28 \\
\hline Hypercholesterolaemia & $30(30)$ & $6(29)$ & 0 & $0.02^{*} \dagger$ \\
\hline Statin therapy & 18(18) & $4(19)$ & 0 & 0.12 \\
\hline Hypertension & $9(9)$ & $5(24)$ & $4(20)$ & 0.11 \\
\hline Smoking & $57(57)$ & $10(53)$ & $2(10)$ & $<0.001^{*} \dagger$ \\
\hline \multicolumn{5}{|l|}{ Cardiac observations } \\
\hline Systolic blood pressure (mm Hg) & $119 \pm 13$ & $124 \pm 11$ & $119 \pm 11$ & 0.28 \\
\hline Diastolic blood pressure $(\mathrm{mm} \mathrm{Hg})$ & $76 \pm 8$ & $76 \pm 8$ & $73 \pm 8$ & 0.22 \\
\hline Resting heart rate (bpm) & $93 \pm 12$ & $79 \pm 11$ & $70 \pm 7$ & $<0.001^{*} \dagger$ \\
\hline $\mathrm{QTc}(\mathrm{ms})$ & $417 \pm 23$ & $394 \pm 22$ & $402 \pm 26$ & $<0.001^{*}$ \\
\hline
\end{tabular}

$0.9(0.2-2.7) \mathrm{mg} / \mathrm{L}, \mathrm{p}=0.045)$ compared with patients receiving non-clozapine treatment and healthy controls, respectively. Lipid profiles revealed similar total and LDL-C levels between the three groups, but higher triglyceride levels were observed in the clozapine group (table 2). No patients had levels of cardiac HsTropT elevated above the laboratory normal range. On post hoc Tukey analysis, there were significant differences in neutrophil: lymphocyte ratio (higher), eosinophil count (lower) and HsTropT (higher) between the clozapine and non-clozapine schizophrenia groups.

\section{Ventricular function}

Comparison of LVEF (figure 1A) and GLS (figure 1B) demonstrated a graded reduction in $\mathrm{LV}$ function across the three groups $(\mathrm{p}<0.001)$ with significant differences between clozapine and non-clozapine schizophrenia groups ( $\mathrm{p}<0.05$, post hoc Tukey test). The fractional shortening, M-mode EF and Simpson's LVEF were all significantly lower for the patients receiving clozapine relative to the other groups (fractional shortening: clozapine vs non-clozapine schizophrenia vs controls 32.1 (24.8-39.1)\% vs $35.9 \quad(29.2-43.7) \%$ vs $36.4 \quad(28.0-51.9) \%, \quad \mathrm{p}=0.001$; M-mode EF 60.7 (49.4-70.4)\% vs 65.7 (58.1-74.7)\% vs 65.6 (54.3-83.5)\%, p=0.003; Simpson's LVEF 58.3 (49.0-67.0)\% vs $\quad 62.2 \quad(55.1-72.9) \%$ vs $64.8 \quad(56.2-79.7) \%, \quad \mathrm{p}<0.001$, respectively). The modified Tei index was significantly higher in clozapine and non-clozapine schizophrenia groups than in controls $(0.59(0.4-0.9)$ vs $0.59(0.4-0.7)$ vs $0.50(0.4-1.0), \mathrm{p}=0.04)$. Of all the methods used to assess LV function, GLS was found to show the greatest relative difference in systolic function between patients receiving clozapine and healthy controls, demonstrating a $17 \%$ difference $(-16.7 \quad(-13.3$ to -20.1$) \%$ vs $-20.2 \quad(-16.6$ to $-25.0) \%, \mathrm{p}<0.001$, respectively).

There was no significant difference in diastolic function between the three groups based on measurements derived from conventional Doppler techniques. However, the peak strain rate in early diastole (E strain rate), a validated index of $\mathrm{LV}$ diastolic function, ${ }^{21}$ was reduced in patients receiving clozapine, implying early diastolic dysfunction (table 3).

The prevalence of more significant cardiomyopathy, defined as Simpson's EF $<50 \%,{ }^{14}$ was $9 \%$ in the 
Table 2 Biochemical parameters

\begin{tabular}{|c|c|c|c|c|}
\hline & Clozapine $(n=100)$ & Non-clozapine $(n=21)$ & Controls $(n=20)$ & p Value (ANCOVA) \\
\hline \multicolumn{5}{|l|}{ Biochemical parameters } \\
\hline Haemoglobin (g/L) & $142 \pm 15$ & $146 \pm 13$ & $148 \pm 10$ & 0.18 \\
\hline White cell count $\left(\times 10^{9} / \mathrm{L}\right)$ & $8.2 \pm 2.6$ & $7.4 \pm 2.4$ & $6.5 \pm 1.5$ & 0.17 \\
\hline Neutrophil count $\left(\times 10^{9} / \mathrm{L}\right)$ & $5.4 \pm 2.2$ & $4.3 \pm 1.8$ & $3.6 \pm 1.0$ & $0.02^{\star} \dagger$ \\
\hline Neutrophil count $<2.0 \times 10^{9} / \mathrm{L}$ & $2(2)$ & $3(14)$ & 0 & 0.02 \\
\hline Neutrophil count $>7.0 \times 10^{9} / \mathrm{L}$ & $20(20)$ & $2(10)$ & 0 & 0.03 \\
\hline Neutrophil : Iymphocyte ratio & $2.9 \pm 1.7$ & $1.9 \pm 1.0$ & $1.7 \pm 0.8$ & $0.01^{*}$ \\
\hline Eosinophil count $\left(\times 10^{9} / \mathrm{L}\right)$ & $0.12 \pm 0.1$ & $0.23 \pm 0.1$ & $0.17 \pm 0.1$ & $0.006 \dagger$ \\
\hline \multicolumn{5}{|l|}{ Fasting lipids (mmol/L) } \\
\hline Total cholesterol & $5.2 \pm 1.1$ & $5.3 \pm 1.0$ & $4.9 \pm 0.7$ & 0.24 \\
\hline Total cholesterol $>5.2 \mathrm{mmol} / \mathrm{L}$ & $40(40)$ & $11(52)$ & $4(20)$ & 0.12 \\
\hline Statin therapy & $18(18)$ & $4(19)$ & 0 & 0.96 \\
\hline Triglycerides & $2.1 \pm 1.3$ & $1.7 \pm 0.7$ & $1.2 \pm 0.6$ & $0.007^{*}$ \\
\hline Total triglyceride $>2.0 \mathrm{mmol} / \mathrm{L}$ & $25(25)$ & $3(15)$ & $1(5)$ & 0.41 \\
\hline HDL-C & $1.2 \pm 0.3$ & $1.1 \pm 0.4$ & $1.4 \pm 0.3$ & 0.05 \\
\hline LDL-C & $3.0 \pm 0.9$ & $3.3 \pm 0.8$ & $3.0 \pm 0.7$ & 0.06 \\
\hline HsCRP (mg/L) & $4.3 \pm 4.3$ & $3.4 \pm 5.4$ & $0.9 \pm 0.8$ & 0.05 \\
\hline NT-proBNP (pmol/L) & $3.4 \pm 4.2$ & $6.4 \pm 9.5$ & $2.8 \pm 2.2$ & 0.05 \\
\hline HsTropT (ng/L) & $9.1 \pm 3.2$ & $6.9 \pm 4.4$ & $5.0 \pm 0.0$ & $0.002 \ddagger \dagger$ \\
\hline Creatine kinase (U/L) & $145 \pm 129$ & $167 \pm 253$ & $129 \pm 52$ & 0.36 \\
\hline Creatine kinase $>250 \mathrm{U} / \mathrm{L}$ & $14(14)$ & $4(19)$ & $2(10)$ & 0.91 \\
\hline HbA1c (\%) & $5.9 \pm 1.0$ & $6.0 \pm 1.0$ & $5.6 \pm 0.9$ & 0.39 \\
\hline \multicolumn{5}{|c|}{$\begin{array}{l}\text { Values are presented as mean } \pm \text { SD or absolute number (\%). } \\
\text { ANCOVA with the following covariates in the modelling: age, alcohol excess, BMI, diabetes, gender, history of CCF, history of ischaemic heart } \\
\text { disease, hypercholesterolaemia and smoking. } \\
{ }^{*} \text { Post hoc Tukey analysis with Bonferroni correction between clozapine and healthy controls, } p \text { value }<0.05 \text {. } \\
\text { †Post hoc Tukey analysis with Bonferroni correction between patients receiving clozapine and non-clozapine patients, p value }<0.05 \text {. } \\
\text { fPost hoc Tukey analysis with Bonferroni correction between non-clozapine and healthy controls, } p \text { value }<0.05 \text {. } \\
\text { ANCOVA, analysis of covariance; BMI, body mass index; CCF, congestive cardiac failure; HbA1c, glycosylated haemoglobin; HDL-C, } \\
\text { high-density lipoprotein cholesterol; HsCRP, high-sensitivity C reactive protein; HsTropT, high-sensitivity Troponin T; LDL-C, low-density } \\
\text { lipoprotein cholesterol; NT-proBNP, N-terminal pro-B-type natriuretic peptide. }\end{array}$} \\
\hline
\end{tabular}

clozapine group, whereas there were no cases in nonclozapine schizophrenia group or in healthy controls but this was not statistically significantly different (ANCOVA, $\mathrm{p}=0.19)$. Remarkably, nearly half $(49 \%)$ of the patients receiving clozapine had GLS values that were below the $95 \%$ CI $(>-16.6 \%)$ of the control group. Among patients in the non-clozapine group, GLS was also impaired relative to healthy controls $(-18.6(-15.7$ to -21.7$) \%$ vs $-20.2(-16.6$ to -25.0$) \%, p=0.03$ post hoc Tukey test), but to a lesser degree than patients receiving clozapine treatment. There were only three patients in the non-clozapine group (14\%) who had impaired GLS below 2 SDs of the control group. There was no significant difference in measures of RV function of the three groups (table 3).

Intraobserver variability for all echocardiographic parameters was less than $8 \%$, which is consistent with the reported literature. ${ }^{18}$ Across the whole study cohort, there was a moderate correlation between LVEF and the direct measure of myocardial function, GLS $(r=-0.54, p<0.001)$. In patients with schizophrenia with LVEF $<50 \%$, the GLS was significantly more impaired than in patients with normal LVEF $(-14.3(-12.1$ to -16.0$) \%$ vs $-17.6(-13.5$ to -20.5$) \%$, respectively, $\mathrm{p}<0.001)$.

\section{Clinical correlation}

Univariate correlations with impaired LV GLS were assessed in the pooled study cohort of patients with schizophrenia (clozapine and non-clozapine treatment), and also separately in only those patients taking clozapine (table 4). Significant correlations included the use of clozapine, neutrophil count, heart rate, body mass index, smoking, diabetes, NT-proBNP, hsCRP, HDL-C and triglycerides. HsTropT did not correlate with the degree of LV dysfunction.

For the pooled cohort of patients with schizophrenia, multilinear regression using backward selection modelling (including only variables with $\mathrm{p} \leq 0.1$ ) identified patients receiving clozapine (standardised $\beta$ coefficient $0.25, \mathrm{p}=0.01$ ), raised heart rate (standardised $\beta$ coefficient $0.21, \mathrm{p}=0.03$ ) and reduced HDL-C levels (standardised $\beta$ coefficient $-0.18, \quad p=0.03$ ) as independent predictors of impaired LV GLS (table 5). When multilinear regression analysis was performed for the group of patients on clozapine treatment, raised neutrophil count (standardised $\beta$ coefficient 0.33, $\mathrm{p}=0.007$ ) and reduced HDL-C level (standardised $\beta$ coefficient -0.27 , $\mathrm{p}=0.02$ ) were the only significant independent predictors of impaired LV GLS (table 6). Heart rate was not a 

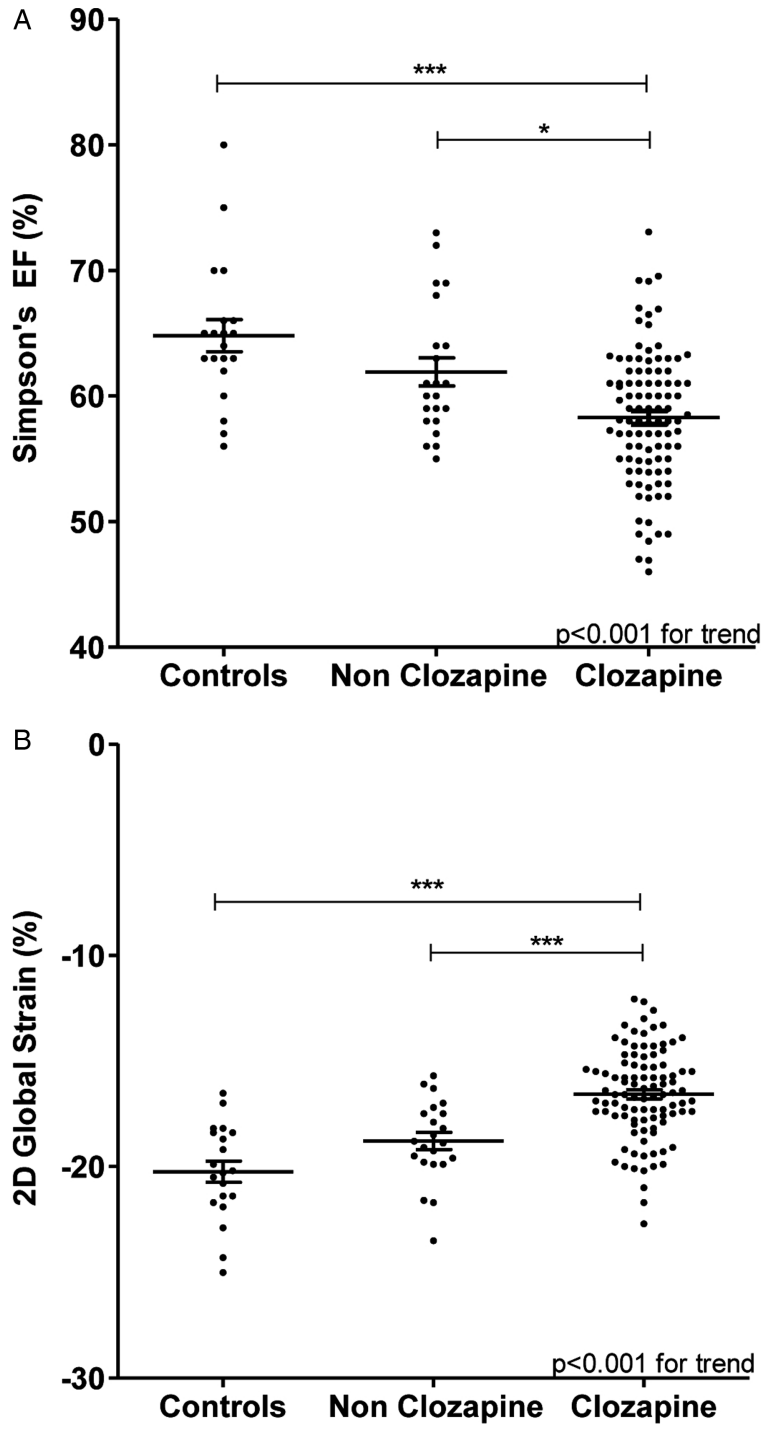

Figure 1 Simpson's biplane ejection fraction $(A)$, and two-dimensional speckle-tracking global longitudinal strain (B) in patients receiving clozapine treatment, non-clozapine treatment and in a healthy control population. *post hoc Tukey test with $p<0.05$; ${ }^{* * *}$ Post hoc Tukey test with $p<0.001$.

significant independent predictor (standardised $\beta$ coefficient $0.13, \mathrm{p}=0.55$ ) in the clozapine group.

\section{DISCUSSION}

The current study represents, to the best of our knowledge, the first systematic study of myocardial function in asymptomatic patients on long-term clozapine treatment for schizophrenia. We have identified mildly impaired cardiac function in patients with schizophrenia, which was more severe in patients taking clozapine, whether measured by conventional parameters of LVEF or by assessment of myocardial function by GLS. We also report for the first time that, in patients taking clozapine, the subclinical impairment of LV function is independently associated with systemic inflammation characterised by neutrophilia and with low HDL-C.
Depending on the definition used to define subclinical cardiomyopathy, between $9 \%(\mathrm{LVEF}<50 \%)$ and $49 \%$ (GLS $>-16.6 \%$, ie, below 2 SDs or the $95 \%$ CI of the normal controls) of patients on long-term clozapine treatment had at least some degree of LV systolic impairment. This may be a conservative estimate given that published normal values for LV GLS are reported to be $-18.6 \pm 0.1 \% .^{18}$ Among non-clozapine-treated patients with schizophrenia, $14 \%$ had impaired GLS based on the above definition. This subclinical LV dysfunction was global (no patients had evidence of segmental dysfunction as is seen after myocardial infarction), was asymptomatic and was not associated with an elevation of HsTropT or with ECG abnormality. There was no evidence of greater dysfunction in those patients having received clozapine for longer duration, suggesting this is not necessarily progressive over time.

The precise mechanism of clozapine-induced muscle injury is not known; however, it is most likely that that the changes are due to direct toxicity of clozapine on the myocardium and not myocarditis as these patients were apparently well and no patient had clinical, ECG or biochemical history to suggest myocarditis. Clozapine is an antagonist of calmodulin, which regulates the contractile response of striated muscles via its effects on calcium uptake, DNA synthesis, neuromuscular transmission and various kinases. Myotoxicity and neurotoxicity have been reported in patients on long-term clozapine treatment based on electromyography, nerve conduction and muscle biopsy studies. ${ }^{22}{ }^{23}$ Raised serum CK levels, a marker of muscle damage, have been reported in 14$78 \%$ of patients on clozapine treatment, independent of dose. $^{22}{ }^{23}$ In our study, 14 of the 100 patients in the clozapine group had raised CK, compared with 2 of 20 controls $(p=n s)$. Similar mechanistic pathways may contribute to clozapine-induced cardiomyopathy. The lack of significant association of NT-proBNP with GLS in multivariate analysis $(\mathrm{p}=0.08)$ may be due to sample size. However, as our echo data found that impairment in LV contractility was a more striking abnormality than chamber dilation with subsequent NT-proBNP release, ${ }^{24}$ the lack of dilation and myocardial stretch explain the relatively modest NT-proBNP release in our cohort.

Our results suggest that the subclinical cardiac dysfunction we have observed with clozapine in this study is not rare and idiosyncratic, as is the incidence of acute myocarditis. Rather, this appears to be a common occurrence affecting a high proportion of patients receiving clozapine, and unlike myocarditis, is unrelated to levels of HsTropT. As some degree of subclinical cardiac impairment may be common in patients receiving clozapine, its detection may not necessarily warrant clozapine cessation. Indeed, premature clozapine cessation can have devastating consequences for effectively treated patients with schizophrenia. Instead, prevention of additional cardiac injury related to recreational drugs such as alcohol and amphetamines, the primary prevention of ischaemic events, and in some circumstances the start of drugs proven useful in the 
Table 3 Echocardiographic parameters

\begin{tabular}{|c|c|c|c|c|}
\hline & Clozapine $(n=100)$ & Non-clozapine $(n=21)$ & Controls $(n=20)$ & p Value (ANCOVA) \\
\hline \multicolumn{5}{|l|}{ Cardiac chamber dimensions } \\
\hline RVEDA $\left(\mathrm{cm}^{2}\right)$ & $9.8 \pm 2.6$ & $11.7 \pm 2.7$ & $10.9 \pm 3.5$ & 0.09 \\
\hline LVEDA $\left(\mathrm{cm}^{2}\right)$ & $25.5 \pm 5.3$ & $29.8 \pm 5.1$ & $27.1 \pm 5.6$ & $0.002^{*}$ \\
\hline RVEDA : LVEDA ratio & $0.39 \pm 0.08$ & $0.37 \pm 0.07$ & $0.40 \pm 0.09$ & 0.48 \\
\hline $\mathrm{RA}$ area $\left(\mathrm{cm}^{2}\right)$ & $12.1 \pm 2.5$ & $11.4 \pm 3.3$ & $12.4 \pm 2.7$ & 0.08 \\
\hline LA area $\left(\mathrm{cm}^{2}\right)$ & $15.0 \pm 3.2$ & $16.0 \pm 4.0$ & $14.5 \pm 2.9$ & 0.93 \\
\hline RA : LA area ratio & $0.83 \pm 0.2$ & $0.72 \pm 0.14$ & $0.87 \pm 0.2$ & 0.04 \\
\hline LVEDV (mL) & $96.5 \pm 27.9$ & $106.5 \pm 30.1$ & $109.5 \pm 26.3$ & 0.49 \\
\hline LVESV (mL) & $38.9 \pm 14.7$ & $39.2 \pm 15.1$ & $38.1 \pm 12.4$ & 0.75 \\
\hline \multicolumn{5}{|l|}{ RV function } \\
\hline RVFAC (\%) & $47.5 \pm 10.3$ & $43.9 \pm 6.7$ & $48.5 \pm 8.9$ & 0.49 \\
\hline TAPSE (cm) & $2.3 \pm 0.3$ & $2.2 \pm 0.2$ & $2.4 \pm 0.2$ & 0.10 \\
\hline PASP $(\mathrm{mm} \mathrm{Hg})$ & $22.7 \pm 6.5$ & $23.0 \pm 3.6$ & $24.3 \pm 4.7$ & 0.94 \\
\hline \multicolumn{5}{|l|}{ LV systolic function } \\
\hline \multicolumn{5}{|l|}{ Conventional parameters } \\
\hline Fractional shortening (\%) & $32.1 \pm 4.8$ & $35.9 \pm 4.0$ & $36.4 \pm 5.7$ & $0.001 \dagger^{*}$ \\
\hline M-mode EF (\%) & $60.7 \pm 6.1$ & $65.7 \pm 4.7$ & $65.6 \pm 6.9$ & $0.003 \dagger^{*}$ \\
\hline Simpson's EF (\%) & $58.3 \pm 5.3$ & $62.2 \pm 5.3$ & $64.8 \pm 5.8$ & $<0.001 \dagger^{\star}$ \\
\hline Simpson's EF <55\% & 27 & 0 & 0 & 0.006 \\
\hline Simpson's EF <50\% & 9 & 0 & 0 & 0.19 \\
\hline LV-modified Tei index & $0.59 \pm 0.13$ & $0.59 \pm 0.08$ & $0.50 \pm 0.15$ & 0.04 \\
\hline \multicolumn{5}{|l|}{ Novel parameters } \\
\hline \multicolumn{5}{|l|}{ 2D speckle systolic strain (\%) } \\
\hline Global longitudinal strain & $-16.7 \pm 2.1$ & $-18.6 \pm 1.6$ & $-20.2 \pm 2.3$ & $<0.001 \dagger \ddagger^{*}$ \\
\hline APLAX & $-16.7 \pm 2.3$ & $-18.1 \pm 1.6$ & $-20.1 \pm 3.0$ & $<0.001 \dagger \ddagger^{\star}$ \\
\hline $4 \mathrm{CH}$ & $-16.6 \pm 2.4$ & $-19.1 \pm 2.3$ & $-20.0 \pm 2.4$ & $0.009 \dagger^{*}$ \\
\hline $2 \mathrm{CH}$ & $-16.9 \pm 2.5$ & $-19.1 \pm 2.3$ & $-20.7 \pm 2.6$ & $<0.001 \dagger^{\star}$ \\
\hline \multicolumn{5}{|l|}{ LV diastolic function } \\
\hline \multicolumn{5}{|l|}{ Conventional parameters } \\
\hline$E / E^{\prime}$ & $7.6 \pm 3.0$ & $8.3 \pm 2.1$ & $7.0 \pm 2.5$ & 0.24 \\
\hline$E^{\prime}(\mathrm{cm} / \mathrm{s})$ & $10.9 \pm 3.9$ & $8.8 \pm 2.4$ & $11.8 \pm 3.9$ & $0.002 \ddagger^{*}$ \\
\hline$A^{\prime}(\mathrm{cm} / \mathrm{s})$ & $10.2 \pm 4.2$ & $10.3 \pm 3.3$ & $8.6 \pm 2.9$ & $0.04 \ddagger \dagger$ \\
\hline $\mathrm{S}^{\prime}(\mathrm{cm} / \mathrm{s})$ & $9.8 \pm 2.8$ & $8.2 \pm 1.6$ & $9.5 \pm 2.6$ & 0.25 \\
\hline \multicolumn{5}{|c|}{ Novel parameters: strain rate $(1 / s)$} \\
\hline \multicolumn{5}{|l|}{ 2D speckle early diastolic (E) } \\
\hline Posterior wall & $1.6 \pm 0.8$ & $1.8 \pm 0.3$ & $1.8 \pm 0.2$ & 0.39 \\
\hline Anteroseptal wall & $1.5 \pm 0.5$ & $1.7 \pm 0.5$ & $1.8 \pm 0.9$ & 0.12 \\
\hline Inferoseptal wall & $1.4 \pm 0.5$ & $1.6 \pm 0.4$ & $1.6 \pm 0.3$ & 0.49 \\
\hline Lateral wall & $1.5 \pm 0.5$ & $1.8 \pm 0.4$ & $1.8 \pm 0.2$ & 0.15 \\
\hline Inferior wall & $1.5 \pm 0.4$ & $1.6 \pm 0.5$ & $1.6 \pm 0.3$ & 0.41 \\
\hline Anterior wall & $1.4 \pm 0.4$ & $1.6 \pm 0.4$ & $1.7 \pm 0.3$ & 0.07 \\
\hline
\end{tabular}

Values are presented as mean \pm SD or absolute number (\%).

ANCOVA with the following covariates in the modelling: age, alcohol excess, BMI, diabetes, family history of mental illness, gender, heart rate, history of CCF, hypercholesterolaemia, hypertension and smoking.

${ }^{*}$ Post hoc Tukey analysis with Bonferroni correction between patients receiving clozapine and non-clozapine patients, $p$ value $<0.05$.

†Post hoc Tukey analysis with Bonferroni correction between clozapine and healthy controls, $p$ value $<0.05$.

$\ddagger$ Post hoc Tukey analysis with Bonferroni correction between non-clozapine and healthy controls, $p$ value $<0.05$.

$2 \mathrm{CH}, 2$ chamber view; 2D, two-dimensional; $\mathrm{A}^{\prime}$, late diastolic/atrial contraction myocardial tissue Doppler velocity measured at lateral mitral annulus; ANCOVA, analysis of covariance; APLAX, apical long axis view; BMI, body mass index; CCF, congestive cardiac failure; E', early diastolic myocardial tissue Doppler velocity measured at lateral mitral annulus; $E / E$ ', ratio of mitral peak velocity of early filling (E) to early diastolic mitral annual velocity (E'); EF, ejection fraction; LA, left atrium; LV, left ventricle; LVEDA, left ventricular end diastolic area; LVEDV, left ventricular end diastolic volume; LVESV, left ventricular end systolic volume; PASP, pulmonary artery systolic pressure; RA, right atrium, RV, right ventricle; RVEDA, right ventricular end diastolic area; RVFAC, right ventricular fractional area change; S', peak systolic myocardial tissue Doppler velocity measured at lateral annulus; TAPSE, tricuspid annular plane systolic excursion.

treatment of cardiac dysfunction in other settings (such as ACE inhibitors and $\beta$ blockers) are likely to be important treatment goals in these patients.

The mechanism of clozapine-induced leucocytosis and neutrophilia is not clear, but increases in granulocyte colony-stimulating factor, tissue necrosis factor- $\alpha$ and interleukin-6 (IL-6) have been proposed.$^{25}$ In patients with schizophrenia, a significant positive correlation had been reported between plasma IL-6 levels and illness duration. ${ }^{26}$ IL-6 is a potent promoter of neutrophil 
Table 4 Univariate correlations with left ventricular 2D speckle-tracking global longitudinal strain

\begin{tabular}{|c|c|c|c|c|}
\hline & \multicolumn{2}{|c|}{ Patients receiving clozapine $(n=100)$} & \multicolumn{2}{|c|}{$\begin{array}{l}\text { All patients with schizophrenia } \\
\text { (clozapine and non-clozapine=121) }\end{array}$} \\
\hline & Correlation coefficient & p Value & Correlation coefficient & p Value \\
\hline Patient receiving clozapine & - & - & 0.35 & $<0.001^{*}$ \\
\hline Heart rate & 0.23 & $0.03^{\star}$ & 0.36 & $<0.001^{*}$ \\
\hline HDL-C level & -0.35 & $<0.001^{\star}$ & -0.22 & $0.02^{*}$ \\
\hline Triglyceride level & 0.20 & $0.04^{\star}$ & 0.20 & $0.03^{*}$ \\
\hline Body mass index & 0.29 & $0.02^{*}$ & 0.21 & $0.03^{*}$ \\
\hline Diabetes & 0.19 & 0.06 & 0.19 & $0.04^{*}$ \\
\hline HsCRP & 0.24 & $0.02^{*}$ & 0.18 & 0.05 \\
\hline Smoking & 0.22 & $0.02^{*}$ & 0.18 & 0.06 \\
\hline Neutrophil count & 0.25 & $0.01^{*}$ & 0.17 & 0.06 \\
\hline Hypercholesterolaemia & 0.16 & 0.12 & 0.15 & 0.11 \\
\hline Eosinophil count & -0.03 & 0.75 & -0.15 & 0.11 \\
\hline NT-proBNP & -0.21 & $0.03^{*}$ & -0.15 & 0.17 \\
\hline $\mathrm{HbA} 1 \mathrm{c}$ & 0.18 & 0.08 & 0.14 & 0.19 \\
\hline HsTropT & 0.01 & 0.89 & 0.07 & 0.45 \\
\hline Age & 0.01 & 0.90 & 0.07 & 0.47 \\
\hline
\end{tabular}

Pearson, Spearman or point-biserial correlation analysis performed based on variable type.

2D, two-dimensional; HbA1c, glycosylated haemoglobin; HDL-C, high-density lipoprotein cholesterol; HsCRP, high-sensitivity C reactive protein; HsTropT, high-sensitivity troponin T; NT-proBNP, N-terminal pro-B-type natriuretic peptide.

release from non-circulating marginal pool into the circulation, resulting in neutrophilia. ${ }^{27}$ Wasti et $a l^{28}$ observed marked leucocytosis and neutrophilia among rats treated with clozapine over a 3-week period. Cytotoxic reactive metabolites of clozapine may play an important role in the pathogenesis of clozapine-induced neutrophilia. Given the correlation we observed between neutrophilia and cardiac dysfunction, it is possible that the same metabolites promote myocardial dysfunction and neutrophilia. There were active smokers among the patients receiving clozapine, and smoking has been shown to be associated with increases in total and differential leucocyte counts. ${ }^{29}$ However, smoking was not an independent predictor of impaired LV GLS on multivariate analysis in the current study and there was no interaction between smoking and neutrophilia in our modelling.

Table 5 Multivariate predictors of left ventricular global longitudinal strain: all patients with schizophrenia $(n=121)$

\begin{tabular}{lcc}
\hline & $\begin{array}{l}\text { Standardised } \\
\text { coefficient } \boldsymbol{\beta}\end{array}$ & $\mathbf{p}$ Value \\
\hline $\begin{array}{l}\text { Patient receiving } \\
\text { clozapine }\end{array}$ & 0.25 & 0.01 \\
Heart rate & 0.21 & 0.03 \\
HDL-C level & -0.18 & 0.03 \\
\hline Backward selection regression model using a p value cut-off of \\
<0.1, $R^{2}=0.22$.
\end{tabular} $\begin{aligned} & \text { Other variables included in the analysis and subsequently } \\
& \text { removed-neutrophil count, NT-proBNP, body mass index, } \\
& \text { HsCRP, age, triglyceride level, smoking and diabetes. } \\
& \text { HDL-C, high-density lipoprotein cholesterol; HsCRP, } \\
& \text { high-sensitivity C reactive protein; NT-proBNP, N-terminal } \\
& \text { pro-B-type natriuretic peptide. }\end{aligned}$

Studies in patients with autoimmune diseases such as systemic lupus erythematosus and rheumatoid arthritis have shown an inverse correlation between HDL-C levels and levels of several proinflammatory markers, with HDL-C having anti-inflammatory properties. ${ }^{30}$ We found that patients on clozapine treatment had reduced HDL-C compared with patients in the non-clozapine group and healthy controls, and reduced HDL-C was an independent predictor of impaired LV contractility. This supports the possibility that a heightened inflammatory state explains the reduced HDL-C levels, and that the subclinical cardiomyopathy of patients with schizophrenia receiving clozapine may be inflammatory in origin. Whether low HDL-C provides unique prognostic or mechanistic information regarding clozapine-related myocardial dysfunction requires further study.

Clozapine-related tachycardia occurs in approximately $25 \%$ of patients, with average heart rate increases $10-$

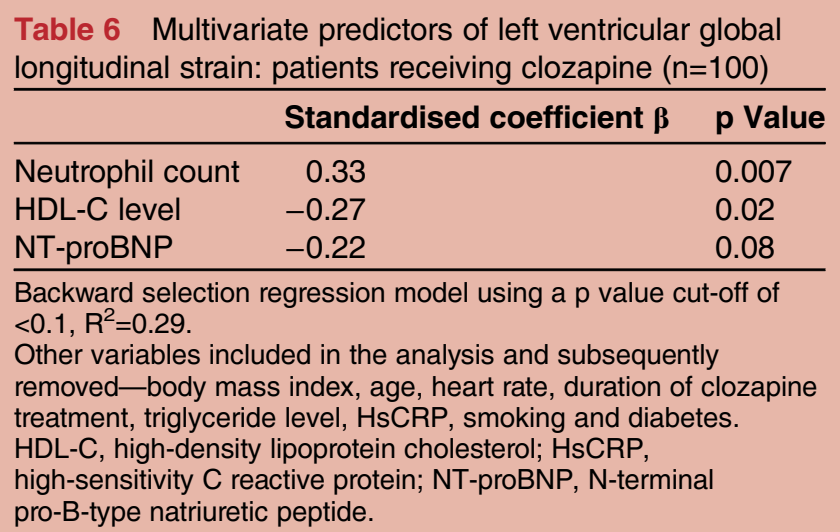


$15 \mathrm{bpm}$ reported. $^{31}$ The mechanism of tachycardia appears to be due to the anticholinergic effects of clozapine, resulting in vagal inhibition. ${ }^{32}$ Persistent tachycardia can result in tachycardia-induced cardiomyopathy. ${ }^{33}$ In our study, although the mean resting heart rate was raised in patients receiving clozapine, it was nonetheless less than $100 \mathrm{bpm}$. In addition, the resting heart rate did not correlate with inflammatory markers such as neutrophil count or hsCRP. Further studies are required to determine the effects of heart rate reduction with pharmacological agents on changes in LV contractility among patients receiving clozapine treatment.

In our study, although patients with schizophrenia receiving non-clozapine antipsychotic medications had less severe impairment in LV systolic function than did patients receiving clozapine, the degree of impairment remained significant when compared with healthy controls in terms of Simpson's EF $(\mathrm{p}=0.03)$ and GLS $(\mathrm{p}=0.009)$. Similar to clozapine, non-clozapine antipsychotic medications have been shown to result in significant weight gain. The development of metabolic syndrome, obesity and diabetes have all been shown to be associated with clinical and subclinical cardiomyopathy. ${ }^{34}{ }^{35}$ However, in a recent study of 2D strain in patients with diabetes and metabolic syndrome, under resting conditions, LV strain and LVEF were identical in patients with and without metabolic syndrome. ${ }^{36}$ This suggests that the degree of impairment we have observed in the present study is unlikely to be adequately explained by associated metabolic changes alone and may have contributions from the direct effects of antipsychotic medications (clozapine and non-clozapine).

The two main goals of cardiac monitoring are to exclude clozapine-induced acute myocarditis and to monitor for long-term cardiomyopathy. The presence of abnormal clinical, echocardiographic or biochemical findings consistent with myocarditis (new onset tachycardia with HR $>120 \mathrm{bpm}$, raised hsCRP, HsTropT, acute deterioration in LVEF and/or systemic symptoms suggestive of myocarditis or cardiac failure) necessitates immediate cardiology review specifically to consider the cessation of clozapine and start of cardioprotective drugs such as $\beta$ blockers and ACE inhibitors. In the absence of myocarditis, the protocol published by Ronaldson $e t a l^{37}$ appears appropriate. Clinical evaluation, ECG and echocardiography are routinely performed prior to treatment and repeated at 3 months, 12 months, 2 years, 5 years and 10 years following the start of clozapine to assess for impaired and/or deteriorating cardiac function. If impaired LV function is identified, an individualised approach is required for each patient in consultation with treating psychiatrists. Treatment options under these circumstances include cardioprotective medications such as ACE inhibitors and $\beta$ blockers, mild gradual reduction of clozapine dose and more frequent review to assess cardiac response to treatment.

\section{LIMITATIONS}

To the best of our knowledge, this study is the single largest contemporary cohort of patients with schizophrenia on long-term clozapine treatment to have undergone detailed echocardiography and biochemical analyses. However, our study does have limitations, including its cross-sectional design. Ideally, our results should be confirmed in long-term, randomised trials comparing clozapine with other antipsychotic treatments. Until recently, there were no consensus recommendations on routine screening for LV dysfunction among patients receiving clozapine, and baseline echocardiography was not mandatory prior to start of clozapine. As a result, we do not have preclozapine treatment echocardiographic data from our patients. The absence of a history of documented ischaemic heart disease, ECG or echocardiographic features of myocardial infarction and the exclusion of cardiac ischaemia with functional assessment in selected patients make prior ischaemic heart disease an unlikely explanation for the $\mathrm{LV}$ dysfunction identified in this young cohort. As this is a cross-sectional study, we cannot exclude the possibility that patients with more severe forms of schizophrenia are in themselves associated with more severe myocardial dysfunction rather than having dysfunction related to clozapine. However, the duration of treatment for schizophrenia was similar in groups taking and not taking clozapine and all patients had been seen in tertiary referral centre for their schizophrenic illness, suggesting that our clozapine and non-clozapine populations were quite severely affected. Finally, our study findings should be extended in future studies by measuring peripheral inflammatory markers including cytokine and lymphocyte subtype analyses to elucidate mechanisms by which subclinical $\mathrm{LV}$ dysfunction is mediated.

\section{CONCLUSION}

Subclinical LV impairment is common in patients with schizophrenia receiving long-term clozapine. This may relate to the degree of systemic inflammation, low plasma HDL-C and/or higher resting heart rate associated with clozapine use. NT-proBNP, HsTropT and hsCRP are not independent predictors of $\mathrm{LV}$ function in patients taking clozapine for long term, and cannot replace the need for routine echocardiographic surveillance.

\section{Author affiliations}

${ }^{1}$ Department of Cardiology, Concord Hospital, The University of Sydney, Sydney, New South Wales, Australia

${ }^{2}$ ANZAC Medical Research Institute, Sydney, New South Wales, Australia ${ }^{3}$ Department of Cardiology, Canterbury Hospital, The University of Sydney, Sydney, New South Wales, Australia

${ }^{4}$ Department of Psychiatry, Croydon Health Centre, Sydney, New South Wales, Australia

${ }^{5}$ National Youth Mental Health Foundation, The University of Sydney, Sydney, New South Wales, Australia

${ }^{6}$ Department of Cardiology, Liverpool Hospital, University of New South Wales, Sydney, New South Wales, Australia 
${ }^{7}$ Department of Cardiology, Royal Prince Alfred Hospital, The University of Sydney, Sydney, New South Wales, Australia

${ }^{8}$ School of Medicine, Notre Dame University, Sydney, Australia

Acknowledgements The authors thank Professor Jenny Peat (consultant statistician) for her assistance in providing statistical advice for the study.

Contributors VC, TY, ACCN and LK designed the study, analysed and interpreted the data. VC, TY and DH extracted the data. VC, ACCN and LK drafted the manuscript. TY, TP, ES, JP, BW, TC, LT and DSC contributed to the critical revision of the manuscript for important intellectual content. VC and LK are guarantors.

Funding This study is supported by the Australian Postgraduate Awards Scholarship from the University of Sydney (Postgraduate Medical Scholarship to $\mathrm{VC}$ ) and a programme grant from the Australian Government National Health and Medical Research Council (DC and LK). Grant number-482820.

Competing interests None.

Ethics approval This study was approved by the Human Ethics Committees of both hospitals.

Provenance and peer review Not commissioned; externally peer reviewed.

Data sharing statement All the data from this study has been presented in this manuscript.

Open Access This is an Open Access article distributed in accordance with the Creative Commons Attribution Non Commercial (CC BY-NC 3.0) license, which permits others to distribute, remix, adapt, build upon this work noncommercially, and license their derivative works on different terms, provided the original work is properly cited and the use is non-commercial. See: http:// creativecommons.org/licenses/by-nc/3.0/

\section{REFERENCES}

1. Volavka J, Czobor P, Sheitman B, et al. Clozapine, olanzapine, risperidone, and haloperidol in the treatment of patients with chronic schizophrenia and schizoaffective disorder. Am J Psychiatry 2002;159:255-62.

2. Meltzer HY, Alphs L, Green Al, et al. Clozapine treatment for suicidality in schizophrenia: International Suicide Prevention Trial (InterSePT). [Erratum appears in Arch Gen Psychiatry.2003 Jul;60 (7):735]. Arch Gen Psychiatry 2003;60:82-91.

3. Tiihonen J, Lonnqvist J, Wahlbeck K, et al. 11-year follow-up of mortality in patients with schizophrenia: a population-based cohort study (FIN11 study). Lancet 2009;374:620-7.

4. Alvir JM, Lieberman JA, Safferman AZ, et al. Clozapine-induced agranulocytosis. Incidence and risk factors in the United States. N Engl J Med 1993;329:162-7.

5. La Grenade L, Graham D, Trontell A. Myocarditis and cardiomyopathy associated with clozapine use in the United States. N Engl J Med 2001;345:224-5.

6. Hummer M, Kurz M, Barnas C, et al. Clozapine-induced transient white blood count disorders. J Clin Psychiatry 1994;55:429-32.

7. Kilian JG, Kerr K, Lawrence C, et al. Myocarditis and cardiomyopathy associated with clozapine. Lancet 1999;354:1841-5

8. [No authors listed]. Effect of enalapril on mortality and the development of heart failure in asymptomatic patients with reduced left ventricular ejection fractions. The SOLVD Investigattors. $N$ Engl J Med 1992;327:685-91.

9. $\mathrm{Ng} \mathrm{AC}$, Delgado $\mathrm{V}$, Bertini $\mathrm{M}$, et al. Findings from left ventricular strain and strain rate imaging in asymptomatic patients with type 2 diabetes mellitus. Am J Cardiol 2009;104:1398-401.

10. Muranaka A, Yuda S, Tsuchihashi K, et al. Quantitative assessment of left ventricular and left atrial functions by strain rate imaging in diabetic patients with and without hypertension. Echocardiography 2009;26:262-71.

11. Jurcut $\mathrm{R}$, Wildiers $\mathrm{H}$, Ganame $\mathrm{J}$, et al. Strain rate imaging detects early cardiac effects of pegylated liposomal Doxorubicin as adjuvant therapy in elderly patients with breast cancer. $J$ Am Soc Echocardiogr 2008;21:1283-9.

12. Antoni ML, Mollema SA, Delgado V, et al. Prognostic importance of strain and strain rate after acute myocardial infarction. Eur Heart $J$ 2010;31:1640-7.
13. American Psychiatric Association. Diagnostic and Statistical Manual of Mental Disorders, Fourth Edition, American Psychiatric Association, Washington, DC, 1994.

14. Lang RM, Bierig M, Devereux RB, et al. Recommendations for chamber quantification: a report from the American Society of Echocardiography's Guidelines and Standards Committee and the Chamber Quantification Writing Group, developed in conjunction with the European Association of Echocardiography, a branch of the European Society of Cardiology. J Am Soc Echocardiogr 2005;18:1440-63.

15. Harada K, Tamura M, Toyono M, et al. Assessment of global left ventricular function by tissue Doppler imaging. Am J Cardiol 2001;88:927-32, A9.

16. Keser N, Yildiz S, Kurtog N, et al. Modified TEl index: a promising parameter in essential hypertension? Echocardiography 2005;22:296-304.

17. Miller D, Farah MG, Liner A, et al. The relation between quantitative right ventricular ejection fraction and indices of tricuspid annular motion and myocardial performance. J Am Soc Echocardiogr 2004;17:443-7.

18. Marwick TH, Leano RL, Brown J, et al. Myocardial strain measurement with 2-dimensional speckle-tracking echocardiography: definition of normal range. JACC CardiovasC Imaging 2009;2:80-4.

19. Yingchoncharoen T, Agarwal S, Popovic ZB, et al. Normal ranges of left ventricular strain: a meta-analysis. J Am Soc Echocardiogr 2013;26:185-91.

20. Negishi K, Negishi T, Hare JL, et al. Independent and incremental value of deformation indices for prediction of trastuzumab-induced cardiotoxicity. J Am Soc Echocardiogr 2013;26:493-8.

21. Leung DY, $\mathrm{Ng} \mathrm{AC}$. Emerging clinical role of strain imaging in echocardiography. Heart Lung Circ 2010;19:161-74.

22. Reznik I, Volchek L, Mester R, et al. Myotoxicity and neurotoxicity during clozapine treatment. Clin Neuropharmacol 2000;23:276-80.

23. Scelsa SN, Simpson DM, McQuistion HL, et al. Clozapine-induced myotoxicity in patients with chronic psychotic disorders. Neurology 1996;47:1518-23

24. Hall C. Essential biochemistry and physiology of (NT-pro)BNP. Eur J Heart Fail 2004;6:257-60.

25. Pollmacher T, Fenzel T, Mullington J, et al. The influence of clozapine treatment on plasma granulocyte colony-stimulating (G-CSF) levels. Pharmacopsychiatry 1997;30:118-21.

26. Monteleone $\mathrm{P}$, Fabrazzo M, Tortorella $\mathrm{A}$, et al. Plasma levels of interleukin-6 and tumor necrosis factor alpha in chronic schizophrenia: effects of clozapine treatment. Psychiatry Res 1997;71:11-17.

27. Hashizume M, Higuchi $Y$, Uchiyama $Y$, et al. IL-6 plays an essential role in neutrophilia under inflammation. Cytokine 2011;54:92-9.

28. Wasti A, Ghani R, Manji MA, et al. Clozapine induced neutrophil cytotoxicity in rats. J Pak Med Assoc 2006;56:62-5.

29. Parry H, Cohen S, Schlarb JE, et al. Smoking, alcohol consumption, and leukocyte counts. Am J Clin Pathol 1997;107:64-7.

30. Haas MJ, Mooradian AD. Inflammation, high-density lipoprotein and cardiovascular dysfunction. Curr Opin Infect Dis 2011;24: 265-72.

31. Safferman A, Lieberman JA, Kane JM, et al. Update on the clinical efficacy and side effects of clozapine. Schizophr Bull 1991;17:247-61.

32. Rechlin T, Claus D, Weis M. Heart rate variability in schizophrenic patients and changes of autonomic heart rate parameters during treatment with clozapine. Biol Psychiatry 1994;35:888-92.

33. Umana E, Solares CA, Alpert MA. Tachycardia-induced cardiomyopathy. Am J Med 2003;114:51-5.

34. Kosmala W, O'Moore-Sullivan T, Plaksej R, et al. Improvement of left ventricular function by lifestyle intervention in obesity: contributions of weight loss and reduced insulin resistance. Diabetologia 2009;52:2306-16.

35. Orhan AL, Uslu N, Dayi SU, et al. Effects of isolated obesity on left and right ventricular function: a tissue Doppler and strain rate imaging study. Echocardiography 2010;27:236-43.

36. Jellis CL, Stanton T, Leano R, et al. Usefulness of at rest and exercise hemodynamics to detect subclinical myocardial disease in type 2 diabetes mellitus. Am J Cardiol 2011;107: 615-21.

37. Ronaldson KJ, Fitzgerald PB, Taylor AJ, et al. A new monitoring protocol for clozapine-induced myocarditis based on an analysis of 75 cases and 94 controls. Aust N Z J Psychiatry 2011;45:458-65. 